\title{
Prognostic factors for surgical resection in patients with multidrug-resistant
} tuberculosis

\author{
H.J. Kim*, C.H. Kang", Y.T. Kim\#, S-W. Sung", J.H. Kim*, S.M. Lee*, C-G. Yoo*, \\ C-T. Lee ${ }^{+}$, Y.W. Kim*, S.K. Han*, Y-S. Shim* and J-J. Yim*
}

ABSTRACT: Although surgical lung resection could improve prognosis in some patients with multidrug-resistant tuberculosis (MDR-TB), there are no reports on the optimal candidates for this surgery. The aim of the present study was to elucidate the prognostic factors for surgery in patients with MDR-TB.

Patients who underwent lung resection for the treatment of MDR-TB between March 1993 and December 2004 were included in the present study. Treatment failure was defined as greater than or equal to two of the five cultures recorded in the final 12 months of treatment being positive, any one of the final three cultures being positive, or the patient having died during treatment. The variables that affected treatment outcomes were identified through univariate and multivariate logistic regression analysis.

In total, 79 patients with MDR-TB were included in the present study. The treatment outcomes of $22(27.8 \%)$ patients were classified as failure. A body mass index $<\mathbf{1 8 . 5} \mathrm{kg} \cdot \mathrm{m}^{-2}$, primary resistance, resistance to ofloxacin and the presence of a cavitary lesion beyond the range of the surgical resection were associated with treatment failure.

Low body mass index, primary resistance, resistance to ofloxacin and cavitary lesions beyond the range of resection are possible poor prognostic factors for surgical lung resection in multidrug-resistant tuberculosis patients.

KEYWORDS: Multidrug-resistant, prognosis, surgery, tuberculosis

M ultidrug-resistant tuberculosis (MDR$\mathrm{TB})$, resistant to at least both isoniazid and rifampicin, poses a serious threat to global health because it requires treatment for a long duration and frequent hospitalisation, and results in a considerable number of mortalities [1]. According to a World Health Organization (WHO) report in 2000, 3.2\% of all new tuberculosis (TB) cases are multidrug resistant (MDR). In Estonia and Latvia, MDR was observed in 14 and $9 \%$ of new TB cases, respectively [2].

The treatment of MDR-TB is difficult as secondline drugs must be used; these are less potent than first-line drugs and are not as well tolerated. Early publications on the treatment response of MDR-TB reported considerable mortality, sometimes as high as $37 \%$ [3]. After the successful introduction of surgical resection of the diseased lung in patients with refractory MDR-TB [4], favourable results among patients with MDR-TB ensued [5-12]. The rates of sputum conversion or of patients who remained negative after surgical resection are as high as $80-98 \%$ [5-12]. Recently, surgical resection, along with the use of new quinolones, has been widely accepted to improve the results of MDR-TB treatment $[13,14]$.

Although surgical resection has been tried and favourable outcomes have been increasingly reported in patients with MDR-TB [5-12], there is no consensus as to which patients should undergo surgical resection. The aim of the current study was to elucidate prognostic factors in patients with MDR-TB refractory to medical treatment who had undergone surgical resection of the diseased lung.

\section{METHODS}

\section{Inclusion criteria and data collection}

The subjects included in the present study were patients who had undergone surgical lung resection for the treatment of MDR-TB at Seoul National University Hospital (Seoul, Republic of Korea), a university-affiliated tertiary referral hospital, between March 1993 and December
AFFILIATIONS

*Division of Pulmonary and Critical Care Medicine, Dept of Internal Medicine and Lung Institute, \#Dept of Thoracic and

Cardiovascular Surgery, Seoul National University College of Medicine, Seoul, and,

"Dept of Thoracic and Cardiovascular Surgery and Respiratory Center, and +Dept of Internal Medicine and Respiratory Center, Seoul National University Bundang Hospital, Gyeonggi-Do, Republic of Korea.

\section{CORRESPONDENCE}

J-J. Yim

Division of Pulmonary and Critical Care Medicine

Dept of Internal Medicine

Seoul National University Hospital

28 Yongon-dong

Chongno-gu

Seoul 110-744

South Korea

Fax: 82220729662

E-mail: yimjj@snu.ac.kr

Received:

February 132006

Accepted after revision:

April 212006

\section{SUPPORT STATEMENT}

This study was funded by grant 04 2005-006-0 from the Seoul National University College of Medicine Research Fund (Seoul, Republic of Korea). 
2004. Although the decision to perform surgical resection was made by individual physicians, the general indication for surgical resection used in the Seoul National University Hospital was MDR-TB refractory to at least 6 months of medical treatment with a main localised lesion. The medical records, microbiology results, other laboratory results, and radiographic examinations of the enrolled patients were reviewed. The protocol of the current study was approved by the Ethical Review Committee of Seoul National University Hospital.

\section{Definitions}

The type of resistance was classified according to the definitions of the WHO [15]. MDR-TB patients without prior treatment with anti-TB drugs were classified as having primary resistance. If the patients had a history of previous anti-TB treatment, they were classified as having an acquired resistance [15]. Body mass index (BMI; $\left.\mathrm{kg} \cdot \mathrm{m}^{-2}\right)$, measured just before the operation, was used in the analysis. The treatment outcomes were classified in accordance with the suggested criteria of LASERSON et al. [16]. Cure was defined as an MDR-TB patient who completed treatment according to the country's protocol and had been consistently culture negative (with at least five results) during the final 12 months of treatment. If only one positive culture was reported during that time, and there was no concomitant clinical evidence of deterioration, a patient may still be considered cured, provided that this positive culture was followed by a minimum of three consecutive negative cultures, taken at least 30 days apart. Treatment failure was defined as greater than or equal to two of the five cultures recorded in the final 12 months being positive, or any one of the final three cultures being positive, as recently suggested. In addition, on analysing treatment outcomes, a patient who died during the course of MDR-TB treatment was included in the treatment failure group.

\section{Statistical analysis}

Data are presented as median values or mean \pm SD. Chisquared test for comparison of categorical variables and unpaired t-test for comparison of continuous variables were applied. Variables analysed include clinical characteristics (age, sex, BMI, type of resistance and drugs used), laboratory results (drug-susceptibility tests, liver-function tests, serum creatinine level and lung function) and radiographic findings. To identify the predictors for the treatment failure after surgical lung resection, multivariate logistic regression models were constructed including age, sex and any variables with a p-value $<0.20$ through univariate analysis. Statistical significance was determined by a $\mathrm{p}$-value $<0.05$.

\section{RESULTS}

\section{Annual number of surgeries and patient baseline characteristics}

Between March 1993 and December 2004, 88 surgical lung resections were carried out at Seoul National University Hospital in 79 patients with MDR-TB refractory to medical treatment. Resections had been performed twice in seven patients and three times in one patient because of persistently positive acid-fast bacilli smear and/or Mycobacterium tuberculosis culture. In 1993, one patient with refractory MDR-TB underwent surgery. The number of surgeries increased to five in 1994 and peaked with 14 surgeries in 2002 (fig. 1). The clinical data of 27 out of the 79 patients were included in an article published in 1999 [9]. All patients were Korean. Among the 79 cases, 48 were male and 31 female. Their median (range) age was 29 (19-60) yrs. The mean BMI was 21.5 and $19.8 \mathrm{~kg} \cdot \mathrm{m}^{-2}$ in the male and female patients, respectively. A total of $24(30.4 \%)$ patients had other underlying diseases. None of the enrolled patients were anti-HIV antibody seropositive. The patients had received a median (range) of six (4-8) anti-TB drugs during a median (range) of 14.5 (9-112) months before the surgery. The $M$. tuberculosis isolates from these patients were resistant to a median (range) of five (2-11) drugs. Drug resistance rates among the 79 patients are summarised in table 1 . At the time of operation, $77(97.5 \%)$ patients were $M$. tuberculosis culture positive. Cavitary lesions were evident in the chest radiographs of $77(97.5 \%)$ patients. Among them, $28(35.4 \%)$ patients had other cavitary lesions beyond the range of the resection. These were as follows: 25 patients had cavitary lesions in the contralateral side to the resected lung and three patients had lesions ipsilateral to the resected lung. In the latter three patients, the cavities could not be completely resected because of limited lung function.

\section{Results of surgical lung resection and subsequent anti-TB chemotherapy}

Among the 79 patients who underwent surgical lung resection, lobectomy was the most common procedure, performed in 44 $(55.7 \%)$ patients. Surgical complications developed in 18 $(22.8 \%)$ patients (table 2$)$. Anti-TB medications were continued for a median (range) of 18 (9-48) months after the surgery. The treatment results were as follows: cure in $57(72.2 \%)$ patients, treatment failure in 21 (26.6\%) patients, and death in one $(1.2 \%)$ patient. The cause of death in the patient in whom right pneumonectomy was performed was respiratory failure caused by worsening TB in the left lung.

\section{Predictors of treatment outcomes}

Several variables were screened for any association with the results of treatment, including surgical lung resection. The variables evaluated included clinical characteristics (age, sex,

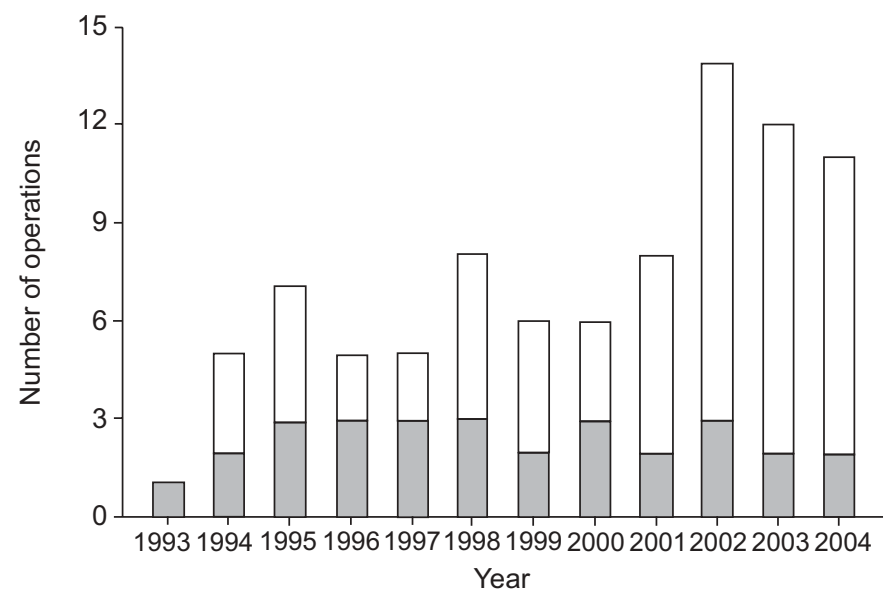

FIGURE 1. Annual numbers of lung resection surgeries in patients with multidrug-resistant tuberculosis (MDR-TB) and their results. A total of 88 operations in 79 patients with MDR-TB took place. $\square$ : successful results; $\square$ : failure. 


\begin{tabular}{|c|c|c|}
\hline TABLE 1 & \multicolumn{2}{|c|}{$\begin{array}{l}\text { Demographic and clinical characteristics of the } \\
79 \text { patients }\end{array}$} \\
\hline Age yrs & & $29(19-60)$ \\
\hline Male/female & & $48: 31$ \\
\hline \multicolumn{3}{|c|}{ Body mass index $\mathrm{kg} \cdot \mathrm{m}^{-2}$} \\
\hline Male & & $21.5(15.4-26.0)$ \\
\hline Female & & $19.8(16.2-25.3)$ \\
\hline Presence of & underlying diseases $\%$ & $24(30.4)$ \\
\hline Diabetes & & $8(10.1)$ \\
\hline Cardiovasc & lar disorders & $7(8.9)$ \\
\hline Chronic live & diseases & $5(6.3)$ \\
\hline COPD or o & er lung diseases & $4(5.1)$ \\
\hline Number of $r$ & sistant drugs & $5(2-11)$ \\
\hline $\begin{array}{l}\text { Resistant tc } \\
\text { nor ofloxa }\end{array}$ & $\begin{array}{l}\text { neither injectables } \\
\text { n }\end{array}$ & $27(34.2)$ \\
\hline Resistant to & injectable & $24(30.4)$ \\
\hline Resistant to & ofloxacin & $10(12.6)$ \\
\hline Resistant tc & injectable and ofloxacin & $18(22.8)$ \\
\hline Duration of & hemotherapy before surgery months & $14.5(9-112)$ \\
\hline \multicolumn{3}{|c|}{ tuberculosis culture before surgery } \\
\hline Number of $\mathrm{c}$ & ugs used before surgery & $6(4-8)$ \\
\hline $\begin{array}{r}\text { Patients rec } \\
\text { containin }\end{array}$ & $\begin{array}{l}\text { iving a regimen } \\
\text { quinolones before surgery }\end{array}$ & $70(88.6)^{\#}$ \\
\hline \multicolumn{3}{|c|}{ Radiographic characteristics } \\
\hline Presence o & cavity & $77(97.5)$ \\
\hline $\begin{array}{l}\text { Presence } 0 \\
\text { of resectio }\end{array}$ & cavity beyond the range & $28(35.4)$ \\
\hline Ipsilatera & to resected lung & $3(3.8)$ \\
\hline Contralat & ral to resected lung & 25 (31.6) \\
\hline Confined to & one lobe & $3(3.8)$ \\
\hline Confined to & one lung & 26 (32.9) \\
\hline Involvemen & of both lungs & $53(67.1)$ \\
\hline
\end{tabular}

Data are presented as median (range) or $n(\%)$, unless otherwise indicated.


patients, ciprofloxacin in three, levofloxacin in 44 and moxifloxacin in two.

BMI, type of resistance and drugs used), the results of microbiological tests and the radiographic findings (table 3 ). Among them, primary resistance $(p<0.001)$, resistance to ofloxacin $(p=0.015)$, and the presence of cavities beyond the range of resection were associated with treatment failure $(p<0.001)$. Multivariate logistic regression analysis involving the variables age, sex and BMI was carried out for association with the results of surgery (table 4$)$. BMI $<18.5 \mathrm{~kg} \cdot \mathrm{m}^{-2}$ $(p=0.043)$, primary resistance $(p<0.001)$, resistance to ofloxacin $(p=0.048)$ and the presence of a cavitary lesion beyond the range of the surgical resection $(p<0.001)$ were associated with treatment failure despite surgical lung resection.

\section{DISCUSSION}

Through the current, retrospective, cohort study, it was demonstrated that lower BMI $(p=0.043)$, primary resistance $(\mathrm{p}<0.001)$, resistance to ofloxacin $(\mathrm{p}=0.048)$, and the presence of a cavitary lesion beyond the range of the resection $(\mathrm{p}<0.001)$ were independently associated with poor treatment outcomes, including surgical resection for treatment of MDR-TB. A lower $\mathrm{BMI}$ and the presence of cavities have been reported to be
TABLE 2 Types of surgery and results of subsequent antituberculosis (TB) chemotherapy in the 79 patients with multidrug-resistant TB

\begin{tabular}{lc} 
Type of pulmonary resection & \\
Wedge resection & $7(8.8)$ \\
Lobectomy & $44(55.7)$ \\
Bilobectomy & $11(13.9)$ \\
Pneumonectomy & $17(21.6)$ \\
Complications of surgery & $18(22.8)$ \\
Bronchopleural fistula with empyema & $4(5.1)$ \\
Prolonged air leak & $7(8.9)$ \\
Infection including pneumonia & $5(6.3)$ \\
Others & $2(2.5)$ \\
Post-operative status & \\
Change in the drug resistance pattern & 5 out of 32 ${ }^{\#}$ \\
Duration of chemotherapy after surgery months & $18(9-48)$ \\
Duration of follow-up after surgery months & $56(9-79)$ \\
Treatment results & \\
Cure & $57(72.2)$ \\
Treatment completed & 0 \\
Death & $1(1.2)$ \\
Treatment defaulted & 0 \\
Treatment failure & $21(26.6)$ \\
Transfer out & 0 \\
\hline & \\
Data are presented as $n$ (\%) or median (range), unless otherwise stated. ${ }^{\#}:$ Pre- \\
and post-operative drug susceptibility test results were available in 32 patients. \\
& \\
& \\
&
\end{tabular}

associated with poor outcomes in patients with MDR-TB [1719]. In addition, use of new quinolones along with surgical resection has been widely accepted to improve the results of MDR-TB treatment $[13,14]$. However, there have been no previous reports of a primary resistance as a poor prognostic factor.

Drug-resistant TB bacilli have been reported as being generally less viable than drug-sensitive bacilli both in vitro and in vivo [20], and have lower transmissibility in most cases [21, 22], although some exceptions have been reported [23, 24]. In this context, the hypothesis that transmitted bacilli with resistance to multidrugs (strains with primary resistance) are phenotypically more virulent than bacilli without definite evidence of transmissibility (strains with acquired resistance), is possible. The report of fatal outcomes from MDR-TB transmitted among family members without definite immune defects supports this hypothesis [25]. However, the observed association between primary resistance and poor prognosis in the current study should be confirmed by future studies enrolling larger numbers of patients with MDR-TB, regardless of surgical resection.

The failure rate of $27.8 \%$ seen in the current study for treatments, including surgical resection, is relatively high compared with other reports [5-12], and could be explained by several factors. First, most patients $(n=77 ; 97.5 \%)$ included in the present study showed positive sputum culture for $M$. tuberculosis at the time of operation. This rate is much higher than the $50 \%$ positive sputum cultures reported among the patients in the study by POMERANTz et al. [7], which showed a 
TABLE 3 Individual predictors for treatment outcomes in multidrug-resistant patients who underwent surgical lung resection

\begin{tabular}{|c|c|c|c|}
\hline Subjects $n$ & 57 & 22 & \\
\hline Male & $36(63.1)$ & $12(54.5)$ & 0.482 \\
\hline $\mathrm{BMI}<18.5 \mathrm{~kg} \cdot \mathrm{m}^{-2}$ & $15(26.3)$ & $10(45.4)$ & 0.101 \\
\hline Primary drug resistance & $6(10.5)$ & $17(77.3)$ & $<0.001$ \\
\hline Quinolone used pre-operatively & $50(87.7)$ & $20(90.9)$ & 0.689 \\
\hline Resistance to ofloxacin & $4(7.0)$ & $6(27.3)$ & 0.015 \\
\hline Resistance to injectables & $18(31.6)$ & $6(27.3)$ & 0.709 \\
\hline Number of resistant drugs $>5$ & $28(49.1)$ & $8(36.4)$ & 0.307 \\
\hline Multilobar involvement & $54(94.7)$ & $22(100)$ & 0.273 \\
\hline Pleuro-pneumonectomy & $13(22.8)$ & $4(7.1)$ & 0.654 \\
\hline
\end{tabular}

Data are presented as mean $\mathrm{SD}$ or $\mathrm{n}(\%)$, unless otherwise stated. BMI: body mass index; $M$. tuberculosis: Mycobacterium tuberculosis.

TABLE 4 Predictors by multivariate logistic analysis for treatment failure in multidrug-resistant tuberculosis patients who underwent surgical lung resection

\begin{tabular}{lcr} 
& Adjusted hazard ratio (95\% Cl) & p-value \\
\hline Age $^{\#}$ & $1.1(0.3-1.8)$ & 0.878 \\
Male & $1.5(0.7-2.9)$ & 0.543 \\
$\mathbf{B M I}<\mathbf{1 8 . 5} \mathbf{~ k g} \cdot \mathbf{m}^{-\mathbf{2}}$ & $2.2(1.4-5.0)$ & 0.043 \\
Primary resistance & $7.1(3.8-16.2)$ & $<0.001$ \\
Resistance to ofloxacin & $2.1(0.9-4.1)$ & 0.048 \\
Presence of cavitary lesion beyond resection & $6.4(3.3-15.7)$ & $<0.001$ \\
\hline
\end{tabular}

Cl: confidence interval; BMI: body mass index. ${ }^{\#}: x+1$ yrs versus $x$ yrs.

$2 \%$ failure rate. Secondly, surgical resection was used aggressively, even in patients with bilateral cavities, because no other treatment options were available. These cases increased the failure rate and showed association with unfavourable outcomes. Thirdly, because all patients with MDR-TB included in the present study had undergone surgical resection because of refractoriness to medical treatment, the results could be unfavourable when compared with those from studies involving various indications, for example, prevention of treatment failure or relapse, or haemoptysis [8].

In conclusion, although the surgical resection of diseased lungs in patients with multidrug-resistant tuberculosis refractory to medical treatment has been accepted as a rescue therapy, clinicians should carefully select the patients for this treatment, giving consideration to possible poor prognostic factors, such as a low body mass index, primary resistance, resistance to ofloxacin, and the presence of cavitary lesions beyond the range of the resection.

\section{ACKNOWLEDGEMENTS}

The authors would like to thank C-H. Lee (Division of HIV and TB control, Korea Center for Disease Control and Prevention, Seoul, Republic of Korea) for his insightful opinion on the manuscript.

\section{REFERENCES}

1 Iseman MD. Treatment of multidrug-resistant tuberculosis. N Engl J Med 1993; 329: 784-791.

2 Dye C, Espinal MA, Watt CJ, Mbiaga C, Williams BG. Worldwide incidence of multidrug-resistant tuberculosis. J Infect Dis 2002; 185: 1197-1202.

3 Goble M, Iseman MD, Madsen LA, Waite D, Ackerson L, Horsburgh CR Jr. Treatment of 171 patients with pulmonary tuberculosis resistant to isoniazid and rifampin. $N$ Engl J Med 1993; 328: 527-532.

4 Pomerantz M, Madsen L, Goble M, Iseman M. Surgical management of resistant mycobacterial tuberculosis and 
other mycobacterial pulmonary infections. Ann Thorac Surg 1991; 52: 1108-1111.

5 Shiraishi Y, Nakajima Y, Katsuragi N, Kurai M, Takahashi N. Resectional surgery combined with chemotherapy remains the treatment of choice for multidrugresistant tuberculosis. J Thorac Cardiovasc Surg 2004; 128: 523-528.

6 Park SK, Lee CM, Heu JP, Song SD. A retrospective study for the outcome of pulmonary resection in 49 patients with multidrug-resistant tuberculosis. Int J Tuberc Lung Dis 2002; 6: 143-149.

7 Pomerantz BJ, Cleveland JC Jr, Olson HK, Pomerantz M. Pulmonary resection for multi-drug resistant tuberculosis. J Thorac Cardiovasc Surg 2001; 121: 448-453.

8 Chiang CY, Yu MC, Bai KJ, Suo J, Lin TP, Lee YC. Pulmonary resection in the treatment of patients with pulmonary multidrug-resistant tuberculosis in Taiwan. Int J Tuberc Lung Dis 2001; 5: 272-277.

9 Sung SW, Kang CH, Kim YT, Han SK, Shim YS, Kim JH. Surgery increased the chance of cure in multi-drug resistant pulmonary tuberculosis. Eur J Cardiothorac Surg 1999; 16: 187-193.

10 van Leuven $M$, De Groot $M$, Shean KP, von Oppell UO, Willcox PA. Pulmonary resection as an adjunct in the treatment of multiple drug-resistant tuberculosis. Ann Thorac Surg 1997; 63: 1368-1372.

11 Kir A, Tahaoglu K, Okur E, Hatipoglu T. Role of surgery in multi-drug-resistant tuberculosis: results of 27 cases. Eur J Cardiothorac Surg 1997; 12: 531-534.

12 Treasure RL, Seaworth BJ. Current role of surgery in Mycobacterium tuberculosis. Ann Thorac Surg 1995; 59: 1405-1407.

13 Chan ED, Laurel V, Strand MJ, et al. Treatment and outcome analysis of 205 patients with multidrug-resistant tuberculosis. Am J Respir Crit Care Med 2004; 169: 1103-1109.

14 Tahaoglu K, Torun $\mathrm{T}$, Sevim $\mathrm{T}$, et al. The treatment of multidrug-resistant tuberculosis in Turkey. $N$ Engl J Med 2001; 345: 170-174.
15 World Health Organization. Guidelines for the management of drug-resistant tuberculosis. Geneva, World Health Organization, 1997.

16 Laserson KF, Thorpe LE, Leimane $\mathrm{V}$, et al. Speaking the same language: treatment outcome definitions for multidrugresistant tuberculosis. Int J Tuberc Lung Dis 2005; 9: 640-645.

17 Yew WW, Chan CK, Chau CH, et al. Outcomes of patients with multidrug-resistant pulmonary tuberculosis treated with ofloxacin/levofloxacin-containing regimens. Chest 2000; 117: 744-751.

18 Leimane V, Riekstina V, Holtz TH, et al. Clinical outcome of individualised treatment of multidrug-resistant tuberculosis in Latvia: a retrospective cohort study. Lancet 2005; 365: 318-326.

19 Mitnick C, Bayona J, Palacios E, et al. Community-based therapy for multidrug-resistant tuberculosis in Lima, Peru. N Engl J Med 2003; 348: 119-128.

20 Ordway DJ, Sonnenberg MG, Donahue SA, Belisle JT, Orme IM. Drug-resistant strains of Mycobacterium tuberculosis exhibit a range of virulence for mice. Infect Immun 1995; 63: 741-743.

21 Godfrey-Faussett $\mathrm{P}$, Sonnenberg $\mathrm{P}$, Shearer SC, et al. Tuberculosis control and molecular epidemiology in a South African gold-mining community. Lancet 2000; 356: 1066-1071.

22 Garcia-Garcia ML, Ponce de Leon A, Jimenez-Corona ME, et al. Clinical consequences and transmissibility of drugresistant tuberculosis in southern Mexico. Arch Intern Med 2000; 160: 630-636.

23 Kruuner A, Hoffner SE, Sillastu H, et al. Spread of drugresistant pulmonary tuberculosis in Estonia. I Clin Microbiol 2001; 39: 3339-3345.

24 Teixeira L, Perkins MD, Johnson JL, et al. Infection and disease among household contacts of patients with multidrug-resistant tuberculosis. Int J Tuberc Lung Dis 2001; 5: 321-328.

25 Sofia M, Maniscalco M, Honore N, et al. Familial outbreak of disseminated multidrug-resistant tuberculosis and meningitis. Int J Tuberc Lung Dis 2001; 5: 551-558. 